Pacific Journal of Mathematic 


\section{ON THE PRINCIPAL FREQUENCY OF A MEMBRANE}

\section{ZeEV NEHARI}

1. Let $D$ denote a simply-connected region in the $x y$-plane whose boundary consists of a finite number of piecewise smooth arcs. If $\lambda$ is the principal frequency of a homogeneous membrane which covers $D$ and is kept fixed at its boundary $C$, then, according to a well-known theorem of Rayleigh [3], $\lambda$ is not smaller than the principal frequency of a circular membrane of equal area and density. This may also be expressed by saying that the homogeneous circular membrane has the lowest principal frequency among all homogeneous membranes of the same mass.

In this paper we shall be concerned with the possible generalizations of Rayleigh's theorem to the case of non-homogeneous membranes. It is clear that no general result of this type is to be expected unless certain restrictions are imposed on the density distribution of the membrane. Indeed, it is easily shown that the principal frequency of a membrane of given mass can be made arbitrarily small if enough of the mass is concentrated in a small area interior to $D$. It is therefore necessary to add conditions which prevent the excessive accumulation of mass at interior points of the membrane. As the following theorem shows, a sufficient condition of this type is the requirement that the density distribution $p(x, y)$ be such that $\log p(x, y)$ is subharmonic, i.e., that the mean value of $\log p(x, y)$ on any circular circumference inside $D$ is not smaller than the value of $\log p(x, y)$ at the center.

THEOREM I. If $\lambda$ is the principal frequency of a membrane of given mass whose density distribution $p(x, y)$ is such that $\log p(x, y)$ is subharmonic, then

$$
\lambda \geqq \lambda_{0},
$$

where $\lambda_{0}$ is the principal frequency of a homogeneous circular membrane of the same mass.

The conclusion of Theorem I will in general not hold if the restriction on $p(x, y)$ is replaced by the somewhat weaker condition that $p(x, y)$ be subharmonic. The following theorem shows, moreover, that - at least in the case of a circular membrane - inequality (1) is reversed if $p(x, y)$ is assumed to be superharmonic.

THEOREM II. If $\lambda$ is the principal frequency of a circular membrane

Received January 3, 1958. This research was supported by the United States Air Force Office of Scientific Research. 
of given mass whose density distribution $p(x, y)$ is superharmonic, then

$$
\lambda \leqq \lambda_{0}
$$

where $\lambda_{0}$ is the principal frequency of a homogeneous circular membrane of the same mass.

Theorems I and II will be proved in $\S \S 2$ and 3 , respectively. In $\S 4$, Theorem $\mathrm{I}$ will be applied to the proof of the following result on homogeneous membranes.

THEOREM III. Let $\alpha$ be an analytic subarc of $C$ which is concave with respect to $D$. If $\Lambda$ denotes the principal frequency of a homogeneous membrane whose boundary is free along $\alpha$ and fixed along $C-\alpha$, then

$$
\Lambda \geqq \Lambda_{0},
$$

where $\Lambda_{0}$ is the principal frequency of a homogeneous semi-circular membrane of equal mass whose boundary is free along the diameter and fixed along the semi-circle.

2. The principal frequency of the membrane with the continuous density distribution $p(x, y)$ is the lowest eigenvalue $\lambda$ of the differential equation

$$
u_{x x}+u_{y y}+\lambda p(x, y) u=0
$$

with the boundary condition $u=0 . \lambda$ may also be defined as the minimum of the Rayleigh quotient

$$
J(U)=\frac{\iint_{D}\left(U_{x}^{2}+U_{y}^{2}\right) d x d y}{\iint_{D} p U^{2} d x d y}
$$

if $U(x, y)$ ranges over the class of functions which vanish on $C$ and for which $U, U_{x}, U_{y}$ are continuous in $D+C$. To prove Theorem I, we have to show that, under the assumptions concerning $p(x, y)$ the integral $\lambda \iint_{D} p d x d y$ attains its minimum in case $D$ is a circular disk and $p(x, y)$ is constant, i.e., we have to demonstrate the inequality

$$
\lambda \iint_{D} p(x, y) d x d y \geqq \pi j_{0}^{2},
$$

where $j_{0}$ is the smallest zero of the Bessel function $J_{0}(r)$.

We denote by $u$ the first eigenfunction of (3). The function $u$ is not zero in $D$, and may be normalized in such a way that $0 \leqq u \leqq 1$ in $D$. We use the symbol $C_{\rho}$ for the level curve, or curves, $u=\rho$ and we 
set $A(\rho)=\iint_{D_{\rho}} p d x d y$, where $D_{\rho}$ is the subset of $D$ at which $u \geqq \rho$. If $C_{\rho}$ consists of $n$ closed Jordan curves (sections of which may coincide), these will be denoted by $C_{\rho, 1}, C_{\rho, 2}, \cdots, C_{\rho, n}$. The proof of (5) will use a symmetrization procedure [3] in which the curve, or curves, $C_{\rho}$ is replaced by a circle about the origin of radius $r$, where $\pi r^{2}=A(\rho)$. If $v$ is the function which takes the value $\rho$ at all points of this circle, and $R$ is defined by $\pi R^{2}=A(0)=\iint_{D} p d x d y$, we shall show that

$$
\iint_{D} p u^{2} d x d y=\int_{0}^{2 \pi} \int_{0}^{R} v^{2} r d r d \theta
$$

and

$$
\iint_{D}\left(u_{x}^{2}+u_{y}^{2}\right) d x d y \geqq \int_{0}^{2 \pi} \int_{0}^{R}\left(v_{x}^{2}+v_{y}^{2}\right) r d r d \theta .
$$

If $J(u)$ and $J(v)$ denote the Rayleigh quotients (3) of $u$ and $v$ for their respective domains of definition, it will follow from (6) and (7) that

$$
\lambda \iint_{D} p d x d y=\pi R^{2} J(u) \geqq \pi R^{2} J(v) .
$$

Since $v(R, \theta)=0, J(v)$ is not smaller than the principal frequency $j_{0}^{2} R^{-2}$ of a homogeneous circular membrane of radius $R$ and density 1 . Theorem I will therefore be proved if (6) and (7) are established.

We denote by $C_{\rho}^{*}$ the level curve $u=\rho-d \rho$, where $d \rho=\varepsilon>0$ and $\varepsilon$ is small. If $d n$ is the length of the piece of the normal to $C$ between $C_{\rho}$ and $C_{\rho}^{*}$, the area between $C_{\rho}$ and $C_{\rho}^{*}$ will be, except for a correction term of order $\varepsilon^{2}$,

$$
d A=\int_{C_{\rho}} p d n d s=\sum_{\nu=1}^{n} \int_{C_{\rho, \nu}} p d n d s
$$

where $s$ is the length parameter on $C_{\rho}$. Since $A(\rho)=\pi r^{2}$, we thus have

$$
2 \pi r d r=\int_{C_{\beta}} p d n d s
$$

By the Schwarz inequality, we have

$$
(d \rho)^{2}\left(\int_{C_{\rho, \nu}} \sqrt{p} d s\right)^{2}=\left(\int_{C_{\rho, \nu}} \frac{d \rho}{d n} \sqrt{p} d n d s\right)^{2} \leqq \int_{C_{\rho, \nu}} p d n d s \int_{C_{\rho, \nu}}\left(\frac{d \rho}{d n}\right)^{2} d n d s
$$

It follows therefore that

$$
(d \rho)^{2} \sum_{\nu=1}^{n}\left(\int_{C_{\rho, \nu}} \sqrt{p} d s\right)^{2} \leqq \int_{C_{\rho}} p d n d s \int_{C_{\rho}}\left(\frac{d \rho}{d n}\right)^{2} d n d s
$$


Since, up to an $\varepsilon^{2}$-correction, $\int_{C \rho}\left(\frac{d \rho}{d n}\right)^{2} d n d s$ is the contribution $D_{\varepsilon}(u)$ of the area between $C_{\rho}$ and $C_{\rho}^{*}$ to the Dirichlet integral on the left-hand side of (7), we thus have, in view of (9).

$$
(d \rho)^{2} \sum_{\nu=1}^{n}\left(\int_{c_{\rho, \nu}} \sqrt{p} d s\right)^{2} \leqq 2 \pi r D_{d \rho}(u) .
$$

On the other hand, the contridution $D_{d r}(v)$ of the circular ring between $r$ and $r+d r$ to the dirichlet integral $D(v)$ is (again with an $\varepsilon^{2}$-correction) $2 \pi r\left(\frac{d \rho}{d r}\right)^{2} d r$. Hence,

$$
D_{d r}(v) \sum_{\nu=1}^{n}\left(\int_{C_{\rho, \nu}} V p d s\right)^{2} \leqq 4 \pi^{2} r^{2} D_{d \rho}(u)
$$

Since $\pi r^{2}=\iint_{D_{\rho}} p d x d y$, this may also be written

$$
D_{d r}(v) \sum_{\nu=1}^{n}\left(\int_{C_{\rho, \nu}} \sqrt{p} d s\right)^{2} \leqq 4 \pi D_{d \rho}(u) \iint_{D_{\rho}} p d x d y
$$

We shall prove presently that, under our assumptions regarding the function $p(x, y)$, the inequality

$$
4 \pi \iint_{G} p d x d y \leqq\left(\int_{\Gamma} \sqrt{p} d s\right)^{2}
$$

holds for any rectifiable Jordan curve $\Gamma$ and the region $G$ bounded by it. If the simply-connected region enclosed by $C_{\rho, \nu}$ is denoted by $D_{\rho, \nu}$, (11) implies that

$$
4 \pi \iint_{D_{\rho}} p d x d y \leqq 4 \pi \sum_{\nu=1}^{n} \iint_{D_{\rho, \nu}} p d x d y \leqq \sum_{\nu=1}^{n}\left(\int_{C_{\rho, \nu}} \sqrt{p} d s\right)^{2}
$$

Combining this with (10), we obtain

$$
D_{d r}(v) \leqq D_{d \rho}(u)
$$

and this entails (7). (6) follows from the fact that, by (9),

$$
\iint_{D^{*}-D} p u^{2} d x d y=\rho^{2} \int_{C_{\rho}} p d n d s+O\left(\varepsilon^{2}\right)=2 \pi r v^{2} d r+O\left(\varepsilon^{2}\right) .
$$

To complete the proof of Theorem I, we have to show that (11) holds for a function $p(x, y)$ which is positive and continuons in a simplyconnected region $G$ and on its boundary $\Gamma$, and which is such that $\log p(x, y)$ is subharmonic. Because of the latter property, we have 
$\log p(x, y) \leqq \sigma(x, y)$ in $G$ if $\sigma(x, y)$ is the harmonic function in $G$ whose boundary values on $\Gamma$ coincide with those of $\log p(x, y)$. Hence, (11) will be proved if we can show that

$$
4 \pi \iint_{G} e^{2 \sigma} d x d y \leqq\left(\int_{\Gamma} e^{\sigma} d s\right)^{2},
$$

where $\sigma(x, y)$ is any harmonic function in $G$ which is continuous in $G+I^{\prime}$. Now $e^{\sigma}=|g(z)|$, where $g(z)$ is a regular analytic function in $G$ which is continuous and does not vanish in $G+\Gamma$. If we set $g(z)=f^{\prime}(z)$, we thus have to show that

$$
4 \pi \iint_{G}\left|f^{\prime}(z)\right|^{2} d x d y \leqq\left(\int_{\Gamma}\left|f^{\prime}(z)\right| d s\right)^{2},
$$

where $f(z)$ is regular in $G$, and $f^{\prime}(z)$ is continuous and does not vanish in $G+\Gamma$.

If $f(z)$ is univalent in $G$, (12) reduces to the isoperimetric inequality

$$
4 \pi \iint_{G^{*}} d \xi d \eta \leqq\left(\int_{\Gamma^{*}}|d w|\right)^{2} \quad(w=\xi+i \eta)
$$

for the region $G^{*}$ (bounded by $\Gamma^{*}$ ) onto which $G$ is mapped by the transformation $w=f(z)$. In the general case we have, by Green's formula,

$$
\iint_{G}\left|f^{\prime}(z)\right|^{2} d x d y=\frac{1}{2 i} \int_{\Gamma} \bar{f} f^{\prime} d z=\frac{1}{2} \int_{\Gamma^{*}}(\xi d \eta-\eta d \xi)
$$

and (12) is seen to be equivalent to the general isoperimetric inequality

$$
2 \pi \int_{\Gamma *}(\xi d \eta-\eta d \xi) \leqq\left(\int_{\Gamma *}|d w|\right)^{2}
$$

proved by Hurwitz [1, p. 97] for arbitrary piecewise smooth closed curves $\Gamma^{*}$ which may be self-intersecting. This completes the proof of Theorem I.

3. We now turn to the proof of Theorem II. Since $p(x, y)$ is superharmonic in the circle $x^{2}+y^{2} \leqq R^{2}$, it follows from a well-known result [2] that

$$
q(r)=\int_{0}^{2 \pi} p(x, y) d \theta \quad\left(x+i y=r e^{i \theta}\right)
$$

is a non-increasing function of $r$ in the interval $[0, R]$. The same is evidently true of its mean value 


$$
\frac{2}{r^{2}} \int_{0}^{r} t q(t) d t
$$

If we set $\tau(r)=\int_{0}^{r} t q(t) d t$, we may therefore conclude that

$$
\tau(r) \geqq \frac{r^{2}}{R^{2}} \tau(R)
$$

If $\lambda$ denotes the lowest eigenvalue of the problem $\Delta v+\lambda p v=0$ with the boundary condition $v=0$ on the circumference $r=R$, we bave

$$
\frac{1}{\lambda} \geqq \frac{\int_{0}^{2 \pi} \int_{0}^{R} p u^{2} r d r d \theta}{\int_{0}^{2 \pi} \int_{0}^{R}\left(u_{x}^{2}+u_{y}^{2}\right) r d r d \theta}
$$

where $u$ is any function which satisfies the boundary and admissibility conditions. In particular, we may take for $u$ the lowest eigenfunction of the problem

$$
\left[r u^{\prime}(r)\right]^{\prime}+\lambda_{0} r u(r)=0, u(R)=u^{\prime}(0)=0 .
$$

This yields

$$
\frac{1}{\lambda} \geqq \frac{\int_{0}^{R} r q(r) u^{2} d r}{2 \pi \int_{0}^{R} r u^{\prime 2} d r} .
$$

In view of the definition of $\tau(r)$, we have

$$
\int_{0}^{R} r q(r) u^{2} d r=\int_{0}^{R} \tau^{\prime}(r) u^{2} d r=-2 \int_{0}^{R} \tau(r) u u^{\prime} d r .
$$

Since $u(r) \geqq 0$ in $[0, R]$, it follows from (15) that $r u^{\prime}(r)$ is a non-increasing function of $r$. Because of $u^{\prime}(0)=0$, we must therefore have $u^{\prime}(r) \leqq 0$ throughout the interval. We thus conclude from (14) and (16) that

$$
\int_{0}^{R} r q(r) u^{2} d r \leqq-2 \frac{\tau(R)}{R^{2}} \int_{0}^{R} r^{2} u u^{\prime} d r=2 \frac{\tau(R)}{R^{2}} \int_{0}^{R} r u^{2} d r .
$$

Hence,

$$
\frac{1}{\lambda} \geqq \frac{\tau(R)}{\pi R^{2}} \frac{\int_{0}^{R} r u^{2} d r}{\int_{0}^{R} r u^{\prime 2} d r}=\frac{\tau(R)}{\pi R^{2} \lambda_{0}}=\frac{\tau(R)}{\pi j_{0}^{2}},
$$

where $j_{0}$ is the first zero of the Bessel function $J_{0}(x)$. Since 


$$
\tau(R)=\int_{0}^{R} r q(r) d r=\int_{0}^{2 \pi} \int_{0}^{R} p r d r d \theta
$$

we finally obtain

$$
\lambda \iint_{D} p d x d y \leqq \pi j_{0}^{2}
$$

and this is equivalent to the assertion of Theorem II.

4. In Theorem III, we are concerned with a boundary value problem of different type. If $\alpha$ is an analytic subarc of $C$, we are considering the problem

$$
\Delta u+A u=0, u=0 \text { on } C-\alpha, \frac{\partial u}{\partial n}=0 \text { on } \alpha .
$$

We shall show that, under the assumption that $\alpha$ is concave with respect to the interior of the membrane, the smallest eigenvalue $\Lambda$ of (17) takes its smallest possible value in the case of a semicircular membrane of the same area, where $\alpha$ coincides with the diameter bounding the membrane. It may be noted that for non-concave $\operatorname{arcs} \alpha$ the assertion of Theorem III will in general not be true; as suitable examples show, $\Lambda$ may in this case be made arbitrarily small.

We introduce the analytic function $f(z)$ which maps the semicircle $|z|<R, \Im\{z\}>0$ conformally onto the region $D$ covered by the membrane, and transforms the segment $-R<z<R$ into the open arc $\alpha$. The value of $R$ may be chosen in such a war that the semicircle has the same area as $D$. Since $\alpha$ is analytic, $f(z)$ will be regular and the mapping will be conformal on the segment $-R<z<R$. Accordingly, the function $v(z)$ defined by $v(z)=u[f(z)]$ will satisfy the boundary condition $\partial v / \partial n=0$ on this linear segment, and (17) is transformed into the problem

$$
\begin{gathered}
\Delta v+\Lambda\left|f^{\prime}(z)\right|^{2} v=0, v=0 \text { for } z=R e^{i \phi}, 0 \leqq \phi \leqq \pi, \\
\frac{\partial v}{\partial n}=0 \text { for }-R<z<R .
\end{gathered}
$$

We now define a function $p(z)$ by $p(z)=\left|f^{\prime}(z)\right|^{2}$ for $|z| \leqq R$, $\{\{z\} \geqq 0$, and $p(z)=\left|f^{\prime}(\bar{z})\right|^{2}$ for $|z| \leqq R, \Im\{z\}<0$. This function is continuous in $|z| \leqq R$, and we may consider the eigenvalue problem

$$
\Delta w+\Lambda^{*} p w=0, w=0 \text { for }|z|=R .
$$

It is easy to see that

$$
\Lambda^{*} \leqq \Lambda,
$$


where $\Lambda$ and $\Lambda^{*}$ are the lowest eigenvalues of (18) and (19), respectively. Indeed, we have

$$
A^{*} \leqq \frac{\iint_{D_{R}}\left(\eta_{x}^{2}+\eta_{y}^{2}\right) d x d y}{\iint_{D_{R}} p \eta^{2} d x d y}
$$

where $D_{R}$ denotes the disk $|z|<R$ and $\eta$ satisfies the boundary and admissibility conditions. If $\eta$ is identified with $v(z)$ in the upper half of $D_{R}$, and with $v(\bar{z})$ in the lower half, these conditions are satisfied and the right-hand side of (21) reduces to $\Lambda$.

The next step is to show that $\log p(z)$ is a subharmonic function in $|z|<R$. This is certainly true in both the upper and the lower open halves of $|z|<R$; indeed, in both these regions $\log p(z)$ is even harmonic. To show that $\log p(z)$ is subharmonic throughout $|z|<R$ it is therefore only necessary to derive the inequality

$$
\log p(x) \leqq \frac{1}{2 \pi} \int_{0}^{2 \pi} \log p\left(x+\varepsilon e^{i \theta}\right) d \theta,
$$

where $x$ is any value such that $-R<x<R$ and $\varepsilon$ is a sufficiently small positive number. Since $p(z)$ is symmetric with respect to the horizontal axis, this is equivalent to

$$
\log p(x) \leqq \frac{1}{\pi} \int_{0}^{\pi} \log p\left(x+\varepsilon e^{i \theta}\right) d \theta,
$$

or, in view of the definition of $p(z)$ in the upper half of the disk $|z|<R$

$$
\log \left|f^{\prime}(x)\right| \leqq \frac{1}{\pi} \int_{0}^{\pi} \log \left|f^{\prime}\left(x+\varepsilon e^{i \theta}\right)\right| d \theta
$$

Since $f(z)$ is regular for $-R<z<R$, we have

$$
\begin{gathered}
\frac{1}{\pi} \int_{0}^{\pi} \log \left|f^{\prime}\left(x+\varepsilon e^{i \theta}\right)\right| d \theta=\Re\left\{\frac{1}{\pi} \int_{0}^{\pi} \log f^{\prime}\left(x+\varepsilon e^{i \theta}\right) d \theta\right\} \\
=\Re\left\{\frac{1}{\pi} \int_{0}^{\pi}\left[\log f^{\prime}(x)+\varepsilon e^{i \theta} \frac{f^{\prime \prime}(x)}{f^{\prime}(x)}+O\left(\varepsilon^{2}\right)\right] d \theta\right\} \\
=\log \left|f^{\prime}(x)\right|-\frac{2 \varepsilon}{\pi} \Re\left\{\frac{1}{i} \frac{f^{\prime \prime}(x)}{f^{\prime}(x)}\right\}+O\left(\varepsilon^{2}\right) .
\end{gathered}
$$

A comparison with (23) shows therefore that (22) will be satisfied for sufficiently small $\varepsilon$ if, and only if, $\Im\left\{f^{\prime \prime}(x) / f^{\prime}(x)\right\}<0$. If $\phi(x)$ is the angle between the tangent to the curve $w=f(x)$ and the positive 
$x$-direction, this is equivalent to $\phi^{\prime}(x)<0$. This condition will therefore be satisfied if, and only if, the curve $w=f(x)$ - that is the arc $\alpha$ - is concave with respect to the interior of $D$. We add that the points at which $\Im\left\{f^{\prime \prime} \mid f^{\prime}\right\}=0$ are either isolated, or else this expression vanishes identically for $-R<x<R$ and $\alpha$ is a linear segment. Evidently, the subharmonicity of $p(z)$ in not destroyed by isolated points of this nature. If $\alpha$ is a linear segment, the assertion of Theorem III follows from Rayleigh's theorem and an elementary symmetry argument.

In accordance with the hypotheses of Theorem III, $\log p(z)$ will thus be subharmonic in $|z|<R$ and we may apply Theorem I, i.e., inequality (5). In view of the definition of $p(z)$, we have

$$
2 A^{*} \iint_{D_{R}}\left|f^{\prime}(z)\right|^{2} d x d y=A^{*} \iint_{|z|<R} p(z) d x d y \geqq \pi j_{0}^{\prime 2}
$$

Taking account of (20) and the fact that $\iint_{D_{R}}\left|f^{\prime}(z)\right|^{2} d x d y$ is the area $A$ of $D$, we obtain

$$
A \Lambda \geqq \frac{\pi}{2} j_{0}^{2}=A j_{0}^{2} R^{-2},
$$

and this is equivalent to the assertion of Theorem III since $j_{0}^{2} R^{-2}$ is the principal frequency of the membrane of density 1 which covers $D_{R}$ and has the indicated boundary conditions.

\section{REFERENCES}

1. R. Courant and D. Hilbert, Methods of mathematical physics, New York, Interscience, 1953.

2. J. E. Littlewood, Lectures on the theory of functions, Oxford University Press, 1944.

3. G. Pôlya and G. Szegö, Isoperimetric inequalities in mathematical physics, Ann. of Math. Studies 27, Princeton University Press, 1951.

CARnegie Institute of TEChNOLOGY 



\section{PACIFIC JOURNAL OF MATHEMATICS}

\section{EDITORS}

\section{H. L. Royden}

Stanford University

Stanford, California

\section{R. A. Beaumont}

University of Washington

Seattle 5 , Washington

\author{
A. L. Whiteman
}

University of Southern California

Los Angeles 7, California

E. G. Straus

University of California

Los Angeles 24, California

\section{ASSOCIATE EDITORS}
E. F. BECKENBACH
A. HORN
L. NACHBIN
G. SZEKERES
C. E. BURGESS
V. GANAPATHY IYER
I. NIVEN
F. WOLF
M. HALL
R. D. JAMES
T. G. OSTROM
E. HEWITT
M. S. KNEBELMAN
M. M. SCHIFFER
K. YOSIDA

\section{SUPPORTING INSTITUTIONS}

\author{
UNIVERSITY OF BRITISH COLUMBIA \\ CALIFORNIA INSTITUTE OF TECHNOLOGY \\ UNIVERSITY OF CALIFORNIA \\ MONTANA STATE UNIVERSITY \\ UNIVERSITY OF NEVADA \\ OREGON STATE COLLEGE \\ UNIVERSITY OF OREGON \\ UNIVERSITY OF SOUTHERN CALIFORNIA
}

\author{
STANFORD UNIVERSITY \\ UNIVERSITY OF UTAH \\ WASHINGTON STATE COLLEGE \\ UNIVERSITY OF WASHINGTON \\ * * * * \\ AMERICAN MATHEMATICAL SOCIETY \\ CALIFORNIA RESEARCH CORPORATION \\ HUGHES AIRCRAFT COMPANY \\ THE RAMO-WOOLDRIDGE CORPORATION
}

Mathematical papers intended for publication in the Pacific Journal of Mathematics should be typewritten (double spaced), and the author should keep a complete copy. Manuscripts may be sent to any of the editors. All other communications to the editors should be addressed to the managing editor, E. G. Straus at the University of California, Los Angeles 24, California.

50 reprints per author of each article are furnished free of charge; additional copies may be obtained at cost in multiples of 50 .

The Pacific Journal of Mathematics is published quarterly, in March, June, September, and December. The price per volume (4 numbers) is $\$ 12.00$; single issues, $\$ 3.50$. Back numbers are available. Special price to individual faculty members of supporting institutions and to individual members of the American Mathematical Society: $\$ 4.00$ per volume; single issues, $\$ 1.25$.

Subscriptions, orders for back numbers, and changes of address should be sent to Pacific Journal of Mathematics, 2120 Oxford Street, Berkeley 4, California.

Printed at Kokusai Bunken Insatsusha (International Academic Printing Co., I.td.), No. 10, 1-chome, Fujimi-cho, Chiyoda-ku, Tokyo, Japan.

PUBLISHED BY PACIFIC JOURNAL OF MATHEMATICS, A NON-PROFIT CORPORATION

The Supporting Institutions listed above contribute to the cost of publication of this Journal, but they are not owners or publishers and have no responsibility for its content or policies. 


\section{Pacific Journal of Mathematics}

\section{Vol. 8, No. 2 \\ April, 1958}

John Herbert Barrett, Second order complex differential equations with a real independent variable ............................ 187

Avner Friedman, Remarks on the maximum principle for parabolic equations and its applications ......................... 201

Richard Robinson Goldberg, An inversion of the Stieltjes transform ....... 213

Olavi Hellman, On the periodicity of the solution of a certain nonlinear integral equation .................................. 219

Gilbert Helmberg, A theorem on equidistribution on compact groups...... 227

Lloyd Kenneth Jackson, Subfunctions and the Dirichlet problem ......... 243

Naoki Kimura, The structure of idempotent semigroups. I ............ 257

Stephen Kulik, A method of approximating the complex roots of equations........................................ 277

Ancel Clyde Mewborn, A note on a paper of L. Guttman.............. 283

Zeev Nehari, On the principal frequency of a membrane ............ 285

G. Pólya and I. J. Schoenberg, Remarks on de la Vallée Poussin means and convex conformal maps of the circle ...................... 295

B. M. Stewart, Asymmetry of a plane convex set with respect to its centroid .......................................... 335

Hans F. Weinberger, Lower bounds for higher eigenvalues by finite difference methods

Edwin Weiss and Neal Zierler, Locally compact division rings ......... 369

Bertram Yood, Homomorphisms on normed algebras ................. 373 